\title{
A preliminary study on mine transient electromagnetic method based on U-shape spiral source
}

\author{
Fei Li, Shangxian Yin, Peng Chen, Demin Liu, Jianxu Pan, Bohao Shi, Xiaoxin Yuan \\ Key Laboratory of Mine Disaster Prevention and Control \\ North China Institute of Science and Technology \\ Beijing 101601, China \\ figo1@163.com
}

\begin{abstract}
A new mine transient electromagnetic method based on U-shape spiral source is proposed. Firstly, the method is introduced. The character of new system is based on a U-shape spiral source which is made up of a U-shape support and the spiral power wire. Furthermore, we analyze the potential advantages of the new method. Finally, we present the observation data measured using the proposed method, which makes a foundation for the following study on data processing and interpretation.
\end{abstract}

Keywords-transient electromagnetic method; U-shape; spiral source; mine

\section{INTRODUCTION}

Transient electromagnetic method (TEM) is a time domain electromagnetic method based on the electromagnetic induction principle. At first, it is mainly used on the surface and in the air with a large configuration. Yu et al. (2007) ${ }^{[1]}$ minimized the configuration and applied it in the roadway. At present, mine transient electromagnetic method (MTEM) has become a key widely applied method in detecting waterbearing structures in mine for its advantages of being sensitive to low resistivity and its convenience. In the MTEM, the center-loop-line configuration and the dipole configuration are two main forms ${ }^{[2]}$. Both of them are based on square-shape loop source.
Though MTEM has made great progress, it has many instinctive limitations ${ }^{[3]}$. (1) The TEM field cannot separate the anomalies in roof and floor (or in front and rear) in whole space. (2) It is easily being influenced by kinds of interference (e.g. industrial electricity, anchors, rails, etc.). (3) It has a detection blind zone because of the self-inductance and mutual inductance. (4) The transmit coil is too large to adapt to the small detection space in roadway sometimes. All the above mentioned limitations have some relations with the squareshape loop source. To overcome the limitations, we propose a new mine transient electromagnetic method based on U-shape spiral source.

\section{THEORY AND METHOD}

A MTEM system is generally made up of the transmit coil, the receive coil, the transmitter and the receiver. Fig. 1 is a traditional MTEM system based on square-shape loop source in dipole configuration. Different from the traditional method, the new system is based on a U-shape spiral source (Fig.2). The receive coil, the transmitter and the receiver in new method is the same as in traditional system, while only the transmit coil is changed into a U-shape form. The U-shape spiral transmit coil is made up of a U-shape support and the spiral power wire, and it looks like a U-shape magnet (Fig.3).

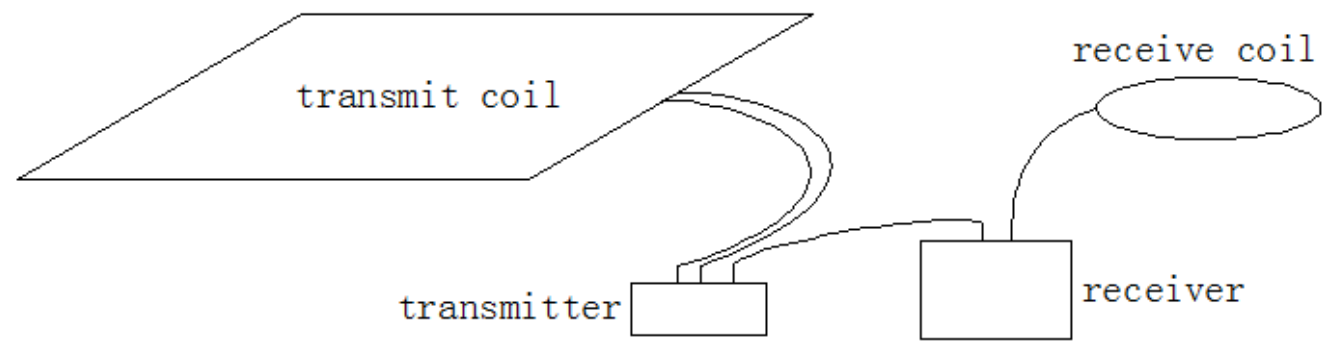

Fig.1. MTEM system based on square-shape loop source 


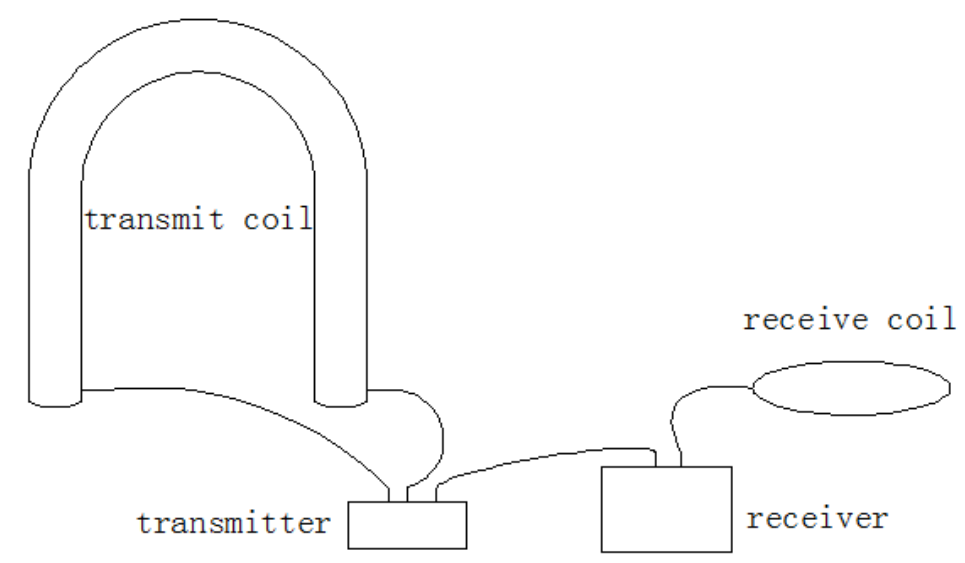

Fig.2. MTEM system based on U-shape spiral source

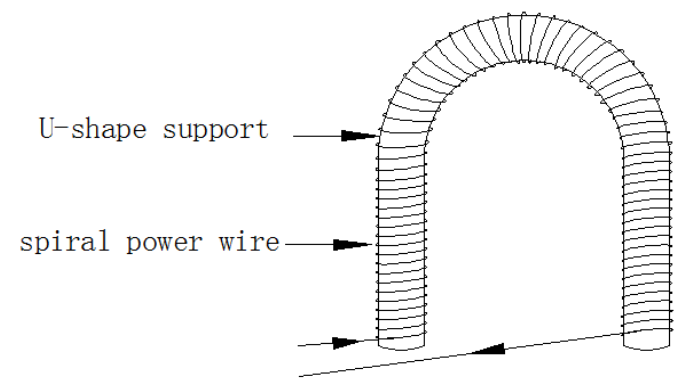

Fig.3. U-shape spiral source transmit coil

Compared with the traditional square-shape loop system, the new method has many potential advantages:

(1)The new system may have stronger anti-interference ability. The detection environment in roadway is complex with all kinds of interference (e.g. industrial electricity, anchors, rails, etc.). The magnetic field of traditional square-shape loop source is divergent(Fig.4), while the magnetic field of U-shape spiral source focuses on a certain zone(Fig.4). Therefore, the interference can be reduced in the new system.

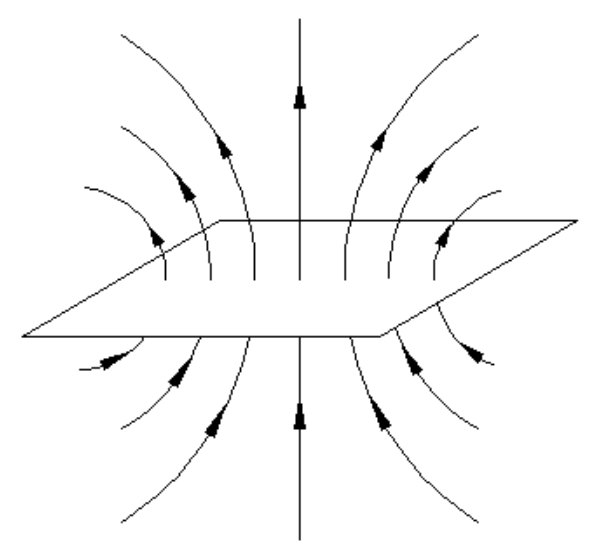

Fig.4. Magnetic field of square-shape loop source 


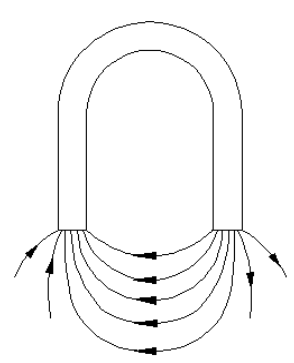

Fig.5. Magnetic field of U-shape spiral source

(2)The new method may accomplish the separation of anomalies in roof and floor (or in front and rear). Fig.5 shows that the magnetic field of U-shape spiral source focuses on the detection direction. Therefore, the secondary magnetic field may mainly diffuse in the detection direction.

(3) The new method may decrease the detection blind zone. The magnetic field of U-shape spiral source is not as divergent as in traditional system, so the mutual inductance between the transmit coil and the receive coil in new system may be reduced.

(4)The new system can be made smaller to adapt to the small detection space in roadway. For example, the width and height of the transmit coil are no more than $1 \mathrm{~m}$ in the following example.

\section{FIELD TEST}

We made a U-shape support with PVC pipes, and retrofitted the PROTEM-47 system to the proposed system based on U-shape spiral source. Then, we accomplished the data collection in the campus. The sample times is from $6.8 \mu$ $\mathrm{s}$ to $6978 \mu \mathrm{s}$, and there are 30 sample times in total. Fig.6 is the measured data using the new system. It can be seen from Fig.6 that the secondary magnetic field measured at the first 21 sample times is good. It makes a good foundation on our following studies on the data processing and interpretation.

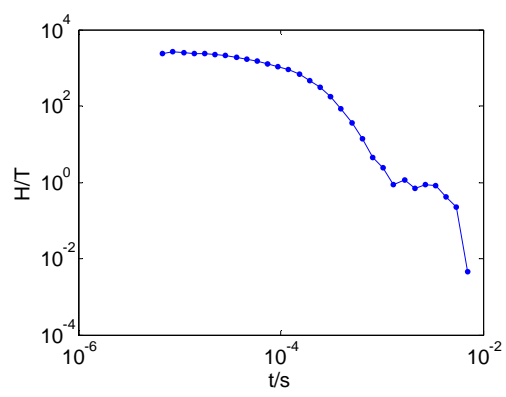

Fig. 6. Measured data using the new TEM method

\section{CONCLUSIONS}

(1) A new mine transient electromagnetic method based on U-shape spiral source is proposed. The new method may have stronger anti-interference ability, and the potential to accomplish the separation of anomalies in roof and floor (or in front and rear).

(2) We accomplished the data collection using the new method. The secondary magnetic field is well measured.

(3) We will study on the data processing and interpretation and verify the potential advantages analyzed in this paper in the future.

\section{ACKNOWLEDGMENT}

The research presented in this paper is supported by the Fundamental Research Funds for the Central Universities (No. 3142015019), Colleges and universities in Hebei province science and technology research project (No. ZC2016036, BJ201603) and Natural Science Foundation of China (Grand No. 51504097).

\section{REFERENCES}

[1] Yu JC, Liu ZX, Liu SC, 2007, Theoretical analysis of mine transient electromagnetic method and its application in detecting water burst structures in deep coal stope: Journal of China Coal Society (in Chinese) , 2007, $8:$ 818-821.

[2] Bai DH, Maxwell AM, Lu J, Wang LF, et al., 2003, Numerical calculation of all-time apparent resistivity for the central loop transient electromagnetic method: Chinese J, Geophys, 26, 3478 - 3484 